\title{
Autonomização da Defesa Europeia por Via da Diferenciação: Cooperação Estruturada Permanente
}

\begin{abstract}
Márcio Jorge Ferreira Guimarães
Consultor de Proteção de Dados. Mestrando em Ciência Jurídico-Internacionais na Faculdade de Direito da Universidade de Lisboa. Licenciado em Ciência Política e Relações Internacionais pela Universidade Fernando Pessoa. Auditor Júnior da Defesa Nacional e Curso Geral de Cibersegurança, PII e DoD Mobile Devices.
\end{abstract}

\section{Resumo}

O Tratado de Lisboa previa o estabelecimento da Cooperação Estruturada Permanente (CEP). Em 2017 um conjunto de 25 Estados-membros decidiu aderir de forma conjunta à CEP de modo a facilitar a cooperação no âmbito da defesa. Para isso, os Estados-membros participantes têm colaborado em diversos projetos e setores da defesa com o objetivo gradual de construção defesa comum.

A AR/VP é a figura que coordena toda uma orquestra, sintonizada com a Agência Europeia de Defesa e o Serviço Europeu de Ação Externa de modo a que os projetos CEP tenham um bom andamento.

Demonstrar-se-á que a RACD, o PDC e o FED são essenciais para que a CEP forneça os ativos necessários para o real impacto nas capacidades de defesa da União.

Portugal demostrou ser prudente, mas assertivo neste processo, podendo fornecer importantes contributos no setor industrial e da investigação.

Palavras-chave: Cooperação Estruturada Permanente; Planos de Desenvolvimento de Capacidades; Revisão Anual Coordenada da Defesa; Fundo de Defesa Europeu.

\author{
Abstract \\ Autonomization of European Defense \\ through Differentiation: Permanent Struc- \\ tured Cooperation
}

The Lisbon Treaty provided for the establishment of Permanent Structured Cooperation (CEP). In 2017, a group of 25 Member States decided join the CEP jointly in order to facilitate defence cooperation. To this end, the participating E-M have collaborated in several defence projects and sectors with the gradual goal of building common defence.

The AR / VP is the figure who coordinates a fully orchestra, in tune with the European Defence Agency and the European External Action Service, so that CEP projects have a good progress. It will be demonstrated that the CARD, the CDP and the EDF are essential for CEP to provide the necessary assets for the real impact on the Union's defence capabilities.

Portugal proved to be careful, but assertive in this process and can provide an important contribution in the industrial and research fields.

Keywords: Permanent Structured Cooperation; Capability Development Plan; Coordinated Annual Review on Defence; European Defence Fund. 


\section{Da Hesitação da Defesa Europeia à Reforma Securitária}

No decorrer das crises económico-financeiras os Estados-membros (E-M) reduziram o seu investimento no setor da defesa, tendo que demostrar uma "resiliência fundamental" para manter os níveis de competitividade e de sustentabilidade na defesa (Barroso, 2016).

Quando a PCSD - antiga PESD e agora integrante da PESC - se tornou efetivamente operacional, houve um esforço intergovernamental para que a União continuasse a conduzir a gestão de crises e conflitos externos, como aconteceu por exemplo nas missões EULEX Kosovo, EUNAVFOR, EUAM Iraque, entre outras ${ }^{1}$.

Os esforços na gestão das políticas de segurança e defesa comportaram um esforço orçamental acrescido dos Estados participantes nos anos de crise, pois em princípio o financiamento das missões e operações no âmbito militar da PCSD não tem o patrocínio orçamental da UE. A despesa sectorial com recursos e capacidades conjeturaram um obstáculo ao investimento no setor da defesa, talvez houvesse uma séria ilusão nas ambições, pois "(...) capabilities are needed on the ground", sendo que os bons projetos e intenções por vezes não passem disso mesmo (Stein, 2016). Em 2016, com Federica Mogherini como AR/VP, foi lançada a "Estratégia Global para a Política Externa e Segurança da União Europeia", aquele que foi um documento inovador, pelo objetivo da autonomização da defesa da UE e, simultaneamente pelo apelo à criação de pontes de diálogo com os parceiros internacionais no desenvolvimento de capacidades e construção de soluções globais ${ }^{2}$. No mesmo ano foi ainda aprovado o plano de execução em matéria de segurança e defesa ${ }^{3}$, que concertava um grau de ambição e cooperação entre E-M mais estreito no âmbito da PCSD, bem como o "Plano de Ação Europeu no Domínio da Defesa", que culminou com a disponibilização do Fundo de Defesa Europeu ${ }^{4}$, assumindo um virar de página definitivo para a defesa e segurança europeia traduzido no investimento e capacitação de meios e recursos mais eficientes.

A cooperação no domínio da construção de uma defesa europeia ${ }^{5}$, é moldada por diferentes camadas de interação com diversas organizações, mormente com a NATO,

1 Note-se que existem diferentes objetivos, modelos de gestão e financiamento entre as missões e operações civis e militares realizadas sob o âmbito da PCSD, tendo que para tal que se observar cada uma cada em pormenor, v. https:/ / eeas.europa.eu/headquarters/headquarters-homepage/area/geo_en.

2 Para mais informações, v. https:/ / eeas.europa.eu/sites/eeas/files/eugs_review_web_0.pdf.

3 Para mais informações, v. https:// www.consilium.europa.eu/media/22460/eugs-implementation-plan-st14392en16.pdf.

4 Para mais informações, v. https:/ / eur-lex.europa.eu/legal-content/PT/TXT/PDF/?uri=CELEX:52016DC0950\&from $=$ en.

5 Note-se, bem diferente e distante de criação de um exército europeu. 
que vem desenvolvendo sinergias de atuação com a UE, destacando a parceria estratégica iniciada em 2001, que se veio desenvolvendo através de linhas de ação prioritárias para ambas as organizações, exemplo disso foram as declarações conjuntas UE-NATO em 2016 e 2018, admitindo um cenário de complementaridade ${ }^{6}$. De forma semelhante, a UE tem vindo a render missões da ONU integradas na PCSD, como aconteceu na Bósnia-Herzegovina ou na República Democrática do Congo. A coincidência das zonas geográficas de atuação para as operações de paz e gestão de crises levou a que a parceria estratégica fosse reforçada para o período compreendido entre 2019 a $2021^{7}$.

A Cooperação Estruturada Permanente - CEP, em inglês PESCO -, prevista no artigo $46 .^{\circ}$, n. $^{\circ} 2$ do Tratado de Lisboa, teria já chamado a atenção dos E-M em 2016, quando o ministro francês e alemão da defesa emitiram uma declaração conjunta na qual defendiam e encorajavam os restantes Estados a avançar para a notificação de criação da CEP, então corroborada pelos Estados do sul e leste europeu (Parlamento Europeu, 2020). A conjuntura internacional ajudou a que este mecanismo fosse acionado, tendo em conta: (i) a imprevisibilidade da eleição do presidente dos EUA, (ii) riscos na vizinhança da UE - ex.: anexação da Crimeia pela Rússia -, (iii) brexit, e, por último, (iv) ações terroristas, todos eles empolaram à intensificam do papel securitário da União.

Em junho de 2017, o Conselho Europeu admitiu "on the need to launch an inclusive and ambitious Permanent Structured Cooperation (PESCO)", apoiando o nível de ambição empregue na Estratégia Global de 2016 (Conselho, 2017).

No dia 13 de novembro de 2017, 23 E-M da UE assinaram uma notificação conjunta dirigida à $\mathrm{AR} / \mathrm{VP}$ comunicando a intenção de participar na CEP, assumindo o cumprimento de compromissos mais vinculativos e o desenvolvimento de projetos colaborativos no domínio da defesa tendo em vista a realização de missões mais exigentes $^{8}$. A 11 dezembro de 2017, tornou-se legalmente vinculativo o compromisso de integração da defesa europeia por 25 EM, incluindo Portugal, estabelecendo

6 A primeira na Cimeira de Varsóvia, em 2016, resultou num compromisso conjunto de execução de 74 medidas concretas, entre as quais em domínios como o setor marítimo/naval, cibersegurança, capacidades de defesa e investigação e indústria de defesa foram elencadas, v. https: / / www.nato.int/cps/en/natohq/official_texts_138829.htm. Em 2018, a declaração reforçou a importância da coerência de ação entre as duas organizações e a necessidade de promover uma cultura de defesa cooperante com os compromissos anteriormente assumidos, v. https: / www. consilium.europa.eu/media/36096/nato_eu_final_eng.pdf.

7 Em 2017, os Estados-membros da UE financiaram mais de 31\% do orçamento para operações de manutenção de paz da ONU.

8 Nesta notificação conjunta não participaram alguns Estado, como Portugal, Irlanda, Dinamarca, Malta e o Reino Unido, v. https:/ / www.consilium.europa.eu/media/31511/171113-pesco-notification.pdf. 
pela primeira vez a Cooperação Estruturada Permanente (CEP), prevista no artigo 42. ${ }^{\circ}$, n. ${ }^{\circ} 6$ e regulada pelo artigo $46 .^{\circ}$ bem como pelo Protocolo n. ${ }^{\circ} 10$ do Tratado da União Europeia (TUE) ${ }^{9}$. Este processo contou com o apoio $a b$ initio do Parlamento Europeu e da Comissão Europeia, procurando a resolução para o problema do subinvestimento de recursos de defesa no seio da UE através da harmonização dos diferentes sistemas de armamento das forças armadas europeias ${ }^{10}$.

Até ao presente, o Conselho aprovou um total de 47 projetos colaborativos de natureza voluntária em três períodos temporais diferentes entre um número variável de EM participantes em cada projeto ${ }^{11}$. Existe uma grande espectativa para verificar até que ponto as capacidades que agora se desenvolvem nos projetos impactam os recursos de defesa europeus, e se esses ativos representam um verdadeiro efeito multiplicador de força naquilo que se pretende efetivamente alcançar, ultima ratio a autonomia estratégica europeia (Sudreau, Efstathiou, e Hannigan, 2019).

As capacidades desenvolvidas no quadro CEP serão da copropriedade do E-M participantes nos projetos, podendo estas ser utilizadas na ótica da interoperabilidade do ponto de vista intra-UE nas missões e operações da PCSD ou, no diálogo extracomunitário com a NATO ou com a ONU.

A segurança interna da União parece ganhar um novo ânimo, a autonomização de uma defesa europeia assente na flexibilidade dos projetos CEP pode levar a cabo importantes reformas das capacidades europeias como o robustecimento da cibersegurança, a investigação tecnológica na segurança marítima e energética, a exploração do sistema espacial ou a mobilidade militar, estes são exemplos de projetos concretos que os E-M concordaram em colaborar de forma a atualizarem e repensarem a forma coma a defesa deve ser operada (Lundin, 2016).

\section{Processo de Governance}

Com a entrada em vigor do Tratado de Lisboa (TL) foram operadas assinaláveis alterações, desde logo a UE apostou num nível de ambição impar no setor da defesa, assumindo de forma explícita o seu compromisso com a segurança internacional, posicionando-se como um produtor de segurança internacional, responsável pelo zelo do bem-estar e paz (artigo 3. ${ }^{\circ}$, n. 5 do TUE) (Koutrakos, 2013; Sari, 2011).

9 Para mais informações, v. https: / / eur-lex.europa.eu/legal-content/EN/TXT/PDF/?uri=CELEX:32017D2315\&from $=$ EN.

10 A UE-28 investe $40 \%$ do que os EUA investem em defesa, mas só gera $15 \%$ das capacidades geradas pelos EUA.

11 As áreas de cada projeto dividem-se por domínio de formação, treino e capacitação, domínio dos sistemas terrestres, domínio marítimo, domínio dos sistemas aéreos, domínio de apoio e capacitação conjunta, domínio da cibersegurança e domínio espacial. 
O TL modificou decisivamente a PCSD, alterando o grau de flexibilidade de ação no que toca às missões civis e militares, bem como na condução efetiva da ação da União no âmbito da gestão civil de crises (Guerra, 2017). A CEP é outro exemplo das inovações introduzidas pelo Tratado de Lisboa, abrindo um espetro de escolhas aos E-M no âmbito da integração da defesa. A principal diferença da CEP para as outras formas de cooperação, como por exemplo a PCSD é a sua natureza juridicamente vinculativa assumida pelos $25 \mathrm{E}-\mathrm{M}$ signatários e a adoção de projetos por maioria qualificada do Conselho, distintivamente das operações e missões no âmbito da PCSD, onde por regra as decisões são tomadas por unanimidade pelo Conselho.

Os 25 E-M não se limitaram a estabelecer a CEP, decidiram integrar um mecanismo ao qual estão obrigados a alcançar metas tangíveis do ponto de vista do reforço e integração securitário da UE. Assim e, uma vez preenchidos os requisitos de admissibilidade estipulados em matéria de capacidades militares no Protocolo n. ${ }^{\circ} 10$, anexo ao TL e estabelecida a CEP, os E-M participantes assumiram uma lista de compromissos comuns e vinculativos que contém 20 compromissos individuais, divididos por cinco áreas descriminadas no artigo $2{ }^{\circ}$ do Protoloco $n .{ }^{\circ} 10$, anexo ao TL, resumidamente: a) orçamental, em função dos objetivos e nível de investimento em defesa; b) equipamento, identificando necessidades militares, reunir, compartilhar e especializar meios e formação; c) operacional, melhorando a interoperabilidade e a projeção de forças; d) recursos, dotando-se de capacidades com o auxílio do "Plano de Desenvolvimento de Capacidades"; e) indústria, participando nos principais programas de aquisição de equipamentos a par da AED.

Os E-M que posteriormente desejem participar na CEP notificam o Conselho e a AR/VP, conforme o descrito no artigo $46 .^{\circ}$, n. $^{\circ} 3$ do TUE. Se a participação do E-M candidato preencher os requisitos estipulados nos artigos $1 .^{\circ}$ e $2 .^{\circ}$ do protocolo $10 .{ }^{\circ}$ do TL, é deliberado por maioria qualificada após consulta do AR/VP e aprovada a sua participação como membro da $\mathrm{CEP}^{12}$. O TL também previu a possibilidade de um Estado participante manifestar a intenção de abandonar a CEP, tendo este que notificar a sua decisão ao Conselho e este toma nota da sua intenção (artigo 46. ${ }^{\circ}$, n. ${ }^{\circ} 5$ do TUE).

E se um Estado participante na CEP não cumprir com os compromissos previamente acordados? Nesse caso, o E-M participante pode ser suspenso, mas previamente a essa ação, é dirigida uma advertência ao E-M participante, constituindo-se um plano de correção do incumprimento, com um calendário definido para consulta individual e tomada de medidas concretas de resposta, nos termos do artigo $46 .^{\circ}$, n. ${ }^{\circ} 4$ do TUE.

12 Note-se que na votação em causa, especificamente neste procedimento, só os Estados-membros participantes tomam parte na votação. 
Na sequência de uma proposta de um Estado participante que tencione fazer parte de uma projeto específico, a AR/VP formula uma recomendação relativa à identificação e à avaliação do projeto CEP tendo por base o parecer militar do Comité Militar da União Europeia (CMUE) e a adoção de decisões e recomendações do Conselho; quando um E-M participante apresente um projeto específico devem informar todos restantes para avaliaram o projeto em tempo útil e, se assim o entenderem associarem-se à submissão da proposta coletiva - artigo $5 .^{\circ}$, n. $^{\circ} 1$ e n. ${ }^{\circ} 2$, Decisão PESC 2017 / 2315 de 11 de dezembro de 2017.

Os Estados participantes num determinado projeto devem acordar entre si as disposições e o âmbito da cooperação bem como a gestão do projeto. É ainda necessário informar regularmente o Conselho sobre o desenvolvimento do projeto de forma adequada. Quanto às decisões e recomendações da CEP, o Conselho adota as mesmas por unanimidade, quando não previstas nos n. ${ }^{\text {s }} 2$ a 5 do artigo $46 .^{\circ}$ do TUE.

Os E-M participantes apresentam os seus Planos de Implementação Nacionais ao Conselho e à AR/VP, nos quais descrevem as medidas tomadas para atingir os objetivos propostos pelas áreas setoriais acima descritas e associadas a cada período ${ }^{13}$. Quanto à monitorização do cumprimento das normas CEP encontram-se previstas no n. ${ }^{\circ}$ 6, do artigo 46. ${ }^{\circ}$ do TUE e na Decisão PESC 2018/909 do Conselho de 25 de junho de 2018, seguindo as normas de voto por unanimidade ou maioria qualificada, conforme aplicável. O procedimento de monitorização é levado a cabo pelos E-M participantes e pela UE através do Conselho, prevendo a existência de sanções para os E-M participantes que não cumpram as metas estipulas, como por exemplo a suspensão como supramencionado. Quando aos critérios de monitorização são transversalmente diligenciados pela chefia da AR/VP, tendo que apresentar um relatório anual de forma detalhada sobre a implementação dos projetos, o grau de execução por parte de cada Estado participante em consonância com os Planos de Implementação Nacionais ${ }^{14}$. O EUMC fornece ao Comitê Político e de Segurança pareceres e recomendações militares sobre o processo anual de avaliação da CEP.

Uma vez por ano o Conselho analisa a continuidade dos E-M participantes e o seu grau de conformidade quanto ao cumprimento dos compromissos mais vinculativos assumidos no Protocolo n. ${ }^{\circ} 10$. No final de cada projeto, pretende-se que a

13 Os Planos de Implementação Nacionais são comunicados ao secretariado da CEP e disponibilizados a todos os restantes Estados participantes.

14 O relatório da AR/VP baseia-se nas informações transmitidas pela AED, em conformidade com o artigo $7 .^{\circ}$, n. ${ }^{\circ} 3$, alínea a), e o SEAE (incluindo o EMUE), em conformidade com o artigo $7 .^{\circ}$, n. $^{\circ}$ 2, alínea a), do Conselho Decisão PESC 2017/2315, de 11 de dezembro de 2017.

Os planos nacionais de execução dos Estados participantes são comunicados anualmente ao Conselho, ao SEAE, à AED e disponibilizados aos restantes E-M participantes. 
copropriedade seja da posse da força unificada que levou a cabo o projeto, ou seja, dos E-M participantes em cada projeto específico.

No final de cada fase (2021 e 2025) será feito um exercício de revisão estratégica, relativamente ao cumprimento dos objetivos previstos para cada fase de forma a atualizar e ajustar se necessário os compromissos (PESCO, 2020).

Chamamos a particular atenção para a possibilidade da participação de Estados terceiros em projetos específicos no âmbito da CEP, regulamentada pelo artigo 9. ${ }^{\circ}$, da Decisão PESC 2017 / 2315 do Conselho de 11 de dezembro de 2017 e pelo ponto 13 da recomendação do Conselho de 6 de março de 2018. No caso, o Conselho ainda não emitiu qualquer Decisão a esta parte, todavia caso venha a ser objeto de apreciação e aprovação do Conselho, a parceria poderá ser regulamentada por via de um acordo administrativo, salvaguardado a autonomia decisória da União. Tendo em conta este cenário, pairam algumas incertezas, como por exemplo, se o grau de exigência para a participação de um Estado terceiro é maior ou menor em relação aos E-M participantes; se os Estados terceiros terão acesso a todas as fases de desenvolvimento do projeto caso venham a ser aceites; se podem solicitar a sua participação em qualquer fase de execução do projeto ou ainda em que medida a sua participação os vincula ou limita em termos de produção industrial. Por agora, sabemos apenas da possibilidade dos E-M participantes poderem realizar acordos administrativos com os Estados terceiros, poderemos vir a ter mais informações caso o Reino Unido venha a solicitar participação em algum projeto, resta esperar para saber quais as condições e termos de participação a definir e divulgar pelo Conselho.

Importante também será frisar o estatuto de Observador, criado pelo artigo $6{ }^{\circ}$ da Decisão PESC 2018 / 909 do Conselho de 25 de junho de 2018, considerando os E-M participantes que queiram solicitar a sua participação enquanto observador num determinado projeto no qual não sejam membros. Os Observadores podem procurar tornar-se membros do projeto numa fase posterior de execução do mesmo, o que poderá ser difícil de concretizar, principalmente em projetos com mais de cinco E-M participantes, uma vez que teria que ser consertado um consenso alargado e, tendo em conta os alinhamentos estratégicos culturais e geográficos de cada E-M poderia comprometer a execução dos projetos em termos temporais (Blockmans e Crosson, 2019, p. 21).

Se observamos os atuais 47 projetos em marcha, destacam-se quatro Estados pelo número de participação em projetos, a saber: Alemanha, França, Itália e Espanha. Este facto poderá demonstrar um sinal do fosso criado pela CEP, contribuindo para coligações de Estados elitistas no seio da UE, pelo seu maior potencial tecnológico e industrial no setor da defesa e ao nível dos clusters ${ }^{15}$. A CEP é apresentada como

15 Lista de Estados europeus com maior capacidade para produzir e exportar armas, v https:// www.sipri.org/databases. 
um mecanismo diferenciador desde o início do seu processo de estabelecimento segregando os E-M segundo critérios de participação exigentes, essa diferenciação foi escolha feita pela UE no TL. Todavia, é necessário acautelar riscos de monopolização da indústria de defesa, nomeadamente através de lobbies e pressões externas que em nada contribuem para a eficiência e sustentabilidade dos projetos podendo originar duplicações de sistemas de armamento de defesa e ameaças, como por exemplo a espionagem industrial ${ }^{16}$. Mais, se o objetivo passa pela harmonização de sistemas de armamento, ou seja, pela definição do menor denominador comum em nome de uma maior eficiência nos gastos, quem irá gerir este padrão de tecnologia ou armamento a ser massificado? O Conselho? É uma questão complexa, à qual os E-M participantes e, principalmente o mercado e indústria de defesa devem estar atentos nos próximos anos.

Recordamos que o Conselho assegura a unidade, a coerência e a eficácia da CEP, sendo que para tal a AR/VP é plenamente associada aos projetos $\mathrm{CEP}$, tendo que apresentar anualmente um relatório suportado pela AED e SEAE. Estas últimas, incluindo o Estado-Maior da União Europeia (EMUE) asseguram de forma conjunta o secretariado da CEP.

O Tratado de Lisboa admitiu um conjunto de meios de natureza político-legal que aspiravam a uma maior consistência em matéria de PCSD, que atualmente está dependente do nível de compromisso dos governos nacionais e da sua vinculação aos compromissos de defesa assumidos, ou seja, da vontade política para a integração gradual da defesa comum.

No que respeita a Portugal as perspetivas são animadoras, participamos em 10 dos 47 projetos já em marcha, assumindo o estatuto de E-M coordenador em dois deles, um ligado à capacitação ao nível da cibersegurança e outro ligado à guerra antissubmarino, mas a sua participação é muito mais abrangente ${ }^{17}$.

16 Estima-se que 26,4 mil milhões de euros sejam desperdiçados todos os anos devido à duplicação de capacidades e às barreiras de aprovisionamento, como resultado são utilizados na Europa seis vezes mais sistemas de defesa do que os EUA.

17 O Estado português participa em 10 projetos no âmbito da CEP, a saber: EU CYBER ACADEMIA AND INNOVATION HUB (EU CAIH), MARITIME (SEMI-) AUTONOMOUS SYSTEMS FOR MINE COUNTERMEASURES (MAS MCM), HARBOUR \& MARITIME SURVEILLANCE AND PROTECTION (HARMSPRO), MARITIME UNMANNED ANTI-SUBMARINE SYSTEM (MUSAS , EUROPEAN SECURE SOFTWARE DEFINED RADIO (ESSOR), CYBER THREATS AND INCIDENT RESPONSE INFORMATION SHARING PLATFORM, STRATEGIC COMMAND AND CONTROL (C2) SYSTEM FOR CSDP MISSIONS AND OPERATIONS, MILITARY MOBILITYM, GEO-METEOROLOGICAL AND OCEANOGRAPHIC (GEOMETOC) SUPPORT COORDINATION ELEMENT (GMSCE) e MATERIALS AND COMPONENTS FOR TECHNOLOGICAL EU COMPETITIVENESS (MAC-EU), v. https: / / pesco.europa.eu/. 


\section{Planeamento, Financiamento e Indústria de Defesa}

O modelo de financiamento previsto para a materialização de projetos integrados no âmbito da CEP deriva do Fundo Europeu de Defesa (FED) e dos orçamentos nacionais de cada E-M participante ${ }^{18}$. O FED terá de ser alinhado de acordo com a Revisão Anual Coordenada da Defesa - RACD, em inglês CARD - e com o Plano de Desenvolvimento de Capacidades - PDC, em inglês CDP - em estreita cooperação com a Agência Europeia de Defesa (AED) (EDA, 2020) ${ }^{19}$.

Na prática, a Comissão lançou dois programas piloto no período de 2017 a 2020 para testar a viabilidade do FED, o seu valor acrescentado para a UE e como meio de preparação para o próximo FED, enquadrado no próximo QFP (2021-2027). O próximo QFP será o maior em toda a história da UE, destinando $8,9 \mathrm{mil} \mathrm{mi-}$ lhões de euros para a vertente das capacidades (atual PEDID), e 4,1 mil milhões de euros para a vertente das capacidades (atual PADR), juntos irão completar o próximo $\mathrm{FED}$, proporcionando incentivos práticos e financeiros à investigação, ao desenvolvimento conjunto e à aquisição de equipamento e tecnologia de defesa. Para a utilização do FED, existem algumas regras/orientações:

- O projeto colaborativo tem de envolver, pelo menos, três entidades elegíveis com sede em pelo menos dois E-M diferentes;

- O nível de financiamento proposto pode ir até $100 \%$ na fase de investigação e de $20 \%$ a $80 \%$ de cofinanciamento para a fase de desenvolvimento;

- Existe a bonificação sobre a forma de taxas de financiamento mais elevadas para promover a participação das PME, inclusive para projetos no âmbito da CEP.

Note-se que a relevância do pacote de financiamento do FED tem como finalidade "promover a competitividade, a eficiência, e a capacitação de inovação da indústria europeia de defesa $(. .$.$) ", com isto a comunidade industrial da defesa europeia deve$

18 Em linha com o artigo 8. ${ }^{\circ}$ da Decisão PESC 2017/ 2315 do Conselho de 11 de dezembro de 2017.

19 Note-se que o Plano de Desenvolvimento de capacidades é atualizado regularmente pela AED, a última revisão foi em 2018, com o objetivo de ajudar os decisores políticos a nível nacional e europeu no que respeita ao desenvolvimento das capacidades de defesa. Da última revisão resultou um conjunto de 11 novas prioridades de desenvolvimento de capacidades para a União, v. https://www.eda.europa.eu/what-we-do/ our-current-priorities / capability-development-plan-.

Com a entrada em vigor do novo FED, a AED e as missões militares no âmbito da PCSD passam a estar sujeitas ao mecanismo de supervisão do Parlamento Europeu com regras de auditoria específicas. 
encontrar um ponto de equilíbrio entre ambições económico-industriais e necessidades estratégicas, para isso a gestão e planeamento dos projetos é crucial.

Quanto à RACD, pode ser comparada à chave-mestra que pode tornar a CEP como o instrumento capaz de edificar a defesa comum da União ${ }^{20}$. A RACD tem a capacidade de situar a UE entre o plano dos objetivos políticos, entenda-se concretização dos projetos CEP e operações e missões no âmbito da PCSD e o Plano de Desenvolvimento de Capacidades que nos revela as principais linhas de orientação naquilo que devem ser os esforços comuns dos E-M. Atualmente, os planos de defesa da UE e da NATO correm o risco de apresentar duplicações e sobreposições de objetivos, processos e capacidades que podem ser consertadas em sede de revisão anual, sob pena do planeamento global de defesa da UE cair no vazio. As ligações entre o PDC, RACD, FED e CEP estão intimamente ligadas e interdependentes da confiança e grau de compromisso dos E-M, por conseguinte, sem planeamento assertivo entre todos estes instrumentos não será possível impactar positivamente a paisagem da defesa europeia e por sua vez, todos estes exercícios individuais não passam de meras boas intenções (ver figura 1).

Figura 1 - A Coherent Approach from Priorities to Impact

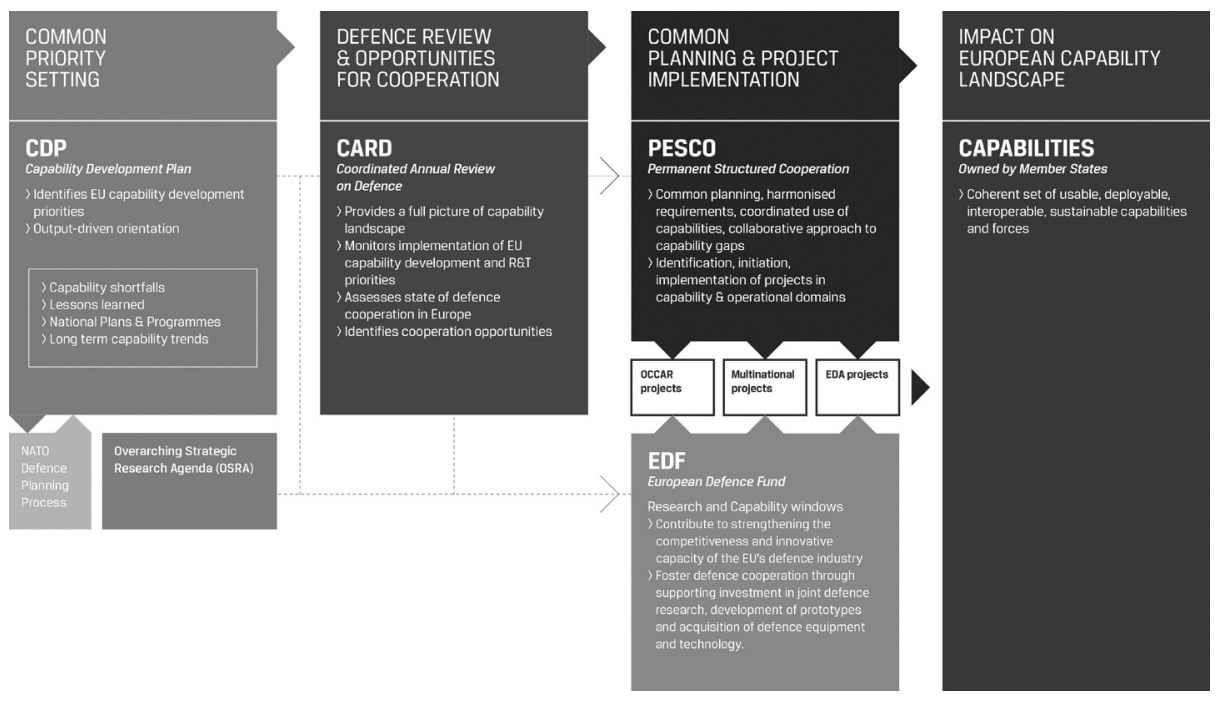

Fonte: EDA

20 Note-se a importância e relevância de documentos orientadores como RACD, resultando de um processo cíclico de avaliação das capacidades de defesa e da convergência estratégica para alcançar os objetivos previamente estabelecidos, contando com a participação da EDA e do SEAE. 
O próximo QFP, conforme o projeto do próximo FED representa um balão de oxigénio para os E-M, promovendo sinergias em projetos de defesa na Europa com o seu cofinanciamento. Não obstante dos valores monetários envolvidos, é necessário enquadrar a dimensão estratégica dos projetos que são apresentados. Não conseguimos identificar um filtro, pelo menos no que toca à limitação da apresentação de projetos numa fase anterior aquela que antecede à passagem do projeto pelo crivo da $\mathrm{AR} / \mathrm{VP}$, existindo um sério risco de se tornar insustentável o número de candidaturas e iniciativas dos E-M participantes a concurso. Seria positivo, por parte da AED ou da AR/VP a emissão de orientações mais objetivas acerca do tipo de projetos que a UE necessita de modo a não despender valores de fundos que poderiam ser orientados e canalizados para determinados projetos que pudessem criar valor acrescentado à UE.

As candidaturas devem observar critérios objetivos em função do Plano de Desenvolvimento de Capacidades e da RACD, tendo que incidir em projetos nos quais hajam lacunas estratégias na UE. Com efeito, para afinar a mira nos projetos efetivamente necessários, poderia ser útil ao Conselho analisar os resultados do Plano de Desenvolvimento de Capacidades em comparação com o grau de implementação das 74 medidas acordadas nas declarações conjuntas com a NATO em Varsóvia e Bruxelas (Blockmans e Crosson, 2019, p.13)

Destacamos a janela de oportunidade para a indústria de defesa nacional ao nível das PME e das microempresas que atuam nesta área e dos seus subsetores, contrariando a lógica heterogénea da BITDE no seio da UE, não compaginável com o objetivo da autonomia industrial de defesa ${ }^{21}$.

Defendemos o investimento na indústria de defesa nacional, não só pelo potencial estratégico para o Estado português, mas também pela capacidade de singrar na vanguarda de projetos de ID \& Research a nível mundial. Recordamos que o investimento nesta indústria representa uma aposta na autonomia de ação do Estado português e na capacidade de Portugal ser um contribuinte importante para a defesa europeia, com capacidade de exportação internacional ${ }^{22}$. Para tal, contamos com a idD - Plataforma das Indústrias de Defesa -, sob a égide do Ministério da Defesa Nacional para potencial as capacidades nacionais de defesa fazendo com que as empresas operantes no mercado português e o próprio Estado se assumam como produtores e exportadores de produtos e serviços no âmbito da economia de defesa, promovendo valor acrescentado e credibilidade internacional.

21 Cerca de 80\% do volume de negócios do setor da defesa estão concertados na Alemanha, França, Itália, Suécia, Espanha e Reino Unido.

22 Os produtos e serviços do setor da defesa são sujeitos a regulamentação e restrições, inclusive a matéria de propriedade intelectual. 
Os eurocéticos da defesa europeia serão os primeiros a criticar à primeira falha da CEP, todavia terão razão se a UE não for capaz de imprimir uma nova coerência face aos instrumentos que dispõe (RACD, PDC e FED), interliga-los e verter para o terreno europeu capacidades que outrora não se vislumbravam. Três anos volvidos desde a sua criação, a CEP revelou alguma dificuldade em se encontrar na complexidade destes instrumentos. Perante este cenário, quanto maior transparência - apresentação detalhada perante os parlamentos nacionais dos Planos de Implementação Nacionais e Planos de Execução - e controlo efetivo a nível nacional e europeu melhor, pois a valorização e credibilização da área da segurança e defesa europeia saem reforçadas e demostram o seu valor no dia a dia dos europeus.

\section{Conclusão}

A CEP tornar-se-á num projeto triunfante se os agentes e instrumentos nacionais e europeus se complementarem de forma a serem coerentes na concretização dos projetos. Os 25 E-M participantes na CEP tem assegurado um reforço e harmonização das suas capacidades de defesa contanto com isso com as empresas numa relação que pretende ser win-win.

A diminuição do número de diferentes sistemas de armamento e aumento da interoperabilidade e competitividade industrial no mercado da defesa da União são os objetivos a atingir, mas espera-se que os projetos que resultam da CEP possam fornecer uma base sólida para a futura defesa comum. A integração intergovernamental dos Estados através das suas Forças Armadas deve procurar corrigir os gaps do passado e harmonizar procedimentos, meios e operações tendo em vista a defesa comum, mesmo que para tal se tenha que optar pela via da diferenciação.

\section{Bibliografia}

Barroso, J., 2016. União Europeia: onde estamos e para onde vamos, Liber Amicorum Fausto de Quadros, Marcelo Rebelo de Sousa e Eduardo Vera Cruz-Pinto (Eds), Vol II, Almedina, pp. 937 e ss.

Blockmans, S. e Crosson, D., 2019. Research Report, Differentiated integration whitin PESCO-clusters and converge in EU defence, N. ${ }^{\circ}$ 2029/04, dezembro de 2019, CEPS, p. 13.

Blockmans, S. e Crosson, D., 2019. Research Report, Differentiated integration whitin PESCO-clusters and converge in EU defence, N. ${ }^{\circ}$ 2029/04, dezembro de 2019, CEPS, p. 21.

Conselho, 2017. European Council meeting 22 and 23 June 2017 - Conclusions. [online]. [consultado em 27/04/2020]. Disponível em: https:/ / www.consilium.europa.eu/media/23985/ 22-23-euco-final-conclusions.pdf 
EDA, 2020. Permanent Structured Cooperation (PESCO). [online]. [consultado em 20/04/2020]. Disponível em: https://www.eda.europa.eu/what-we-do/our-current-priorities/permanent-structured-cooperation-(PESCO)

Guerra, A., 2017. Manual de Direito da União Europeia - Após o Tratado de Lisboa, 2. ${ }^{\text {Ed}}$ Ed., Almedina, páginas 371-177.

Koutrakos, P., 2013. The EU Common Security and Defense Policy, European Journal of International Law, Julia Schmidt, Oxford, Oxford University Press.

Lundin, L., 2016. The Comprehensive Approach and European Union External Action: Focus on the EU Official. Nação e Defesa, The Eu Comprehensive Approach, N. ${ }^{\circ} 144$, pp. 77-87.

Parlamento Europeu, 2020. Permanent Structured Cooperation: national perspectives and state of play. [online]. [consultado em 23/04/2020]. Disponível em: https:/ / www.europarl.europa.eu/RegData/etudes/STUD/2017/603842/EXPO_STU(2017)603842_EN.pdf

PESCO, 2020. Pesco Participating Member States. [online]. [consultado em 22/04/2020]. Disponível em: https:/ / pesco.europa.eu/about/

Sari, A., 2011. International Law Aspects of the EU’s Security and Defense Policy, With a Particular Focus on the Law of Armed Conflict. European Law Review, Fredrick Naert (ed), pp. 451-453.

Stein, T., 2016. The Common Security and Defense Policy - Europe as a Global Actor? In: Liber Amicorum Fausto de Quadros, Marcelo Rebelo de Sousa e Eduardo Vera Cruz-Pinto (Eds), Vol II, Coimbra, p. 901.

Sudreau, L., Efstathiou, Y. e Hannigan, C., 2019. Keeping the momentum in European defence collaboration: an early assessment of PESCO implementation, maio de 2019, The International Institute for Strategic Studies. 